\title{
Plasma formulary interactive
}

\section{E. Stambulchik ${ }^{1}$ and Y. Maron}

Faculty of Physics, Weizmann Institute of Science,

Rehovot 76100, Israel

E-mail: Evgeny.Stambulchik@weizmann.ac.il

ABSTRACT: We present an interactive, Web-based application that provides a comfortable access to evaluating, comparing, and plotting of a multitude of different plasma and atomic physics parameters, grouped by the unit dimensions. A special attention is devoted to the Stark broadening which is calculated with a good accuracy for transitions between degenerate atomic levels. The list of the entities calculated encompasses over one hundred entries and is easily expandable. The application is built using open-source components and is compatible with a majority of modern browsers.

KEYWORDS: Simulation methods and programs; Plasma diagnostics - charged-particle spectroscopy; Plasma diagnostics - interferometry, spectroscopy and imaging

\footnotetext{
${ }^{1}$ Corresponding author.
} 


\section{Contents}

1 Introduction 1

2 Models and assumptions 2

3 Formulary 2

3.1 Atomic units 2

3.2 Basic entities 3

3.3 Waves 4

3.4 Collisions 5

3.5 Spectroscopy 5

3.6 Radiation 8

4 User's guide $\quad 8$

\section{Introduction}

Plasma physics is a rapidly developing discipline integrating practically all subjects of modern fundamental and applied physics, including electrodynamics, kinetic theory, and atomic, molecular, and optical physics. There are many excellent texts devoted to different subjects of plasma physics, however, because of the vastness of material, it is hardly possible to address all of it under a single textbook cover. Furthermore, thorough studies focused on a specific matter may unintentionally make finding a simple general (even though not highly accurate) estimation difficult for a casual reader. Filling in the gap are compilations of formulae from different fields of plasma physics, allowing for calculations of a multitude of relevant entities using straightforward numerical expressions. One such a compilation is the venerable NRL Plasma Formulary by J.D. Huba [1], originally inspired by D.L. Book. Handbooks by A. Anders [2] and D.A. Diver [3] are providing more extensive coverage, each at a somewhat different perspective.

The purpose of the present work is to extend usability of the existing plasma formularies. To this end, a Web-based computer application was designed that allows for (i) calculating miscellaneous plasma physics entities in an interactive manner, (ii) comparing several pre-selected ones (having, evidently, the same dimensionality), and (iii) plotting resulting values as a function of one of the plasma parameters, e.g., the electron density. The application is built using open-source components and is itself released under the GNU General Public License [4] terms. Furthermore, each entity is linked to the corresponding on-line Wikipedia [5] article, thus encouraging practitioners in the fields of atomic and plasma physics to check, revise, and contribute in other ways to the world-largest free encyclopedia. 


\section{Models and assumptions}

In the calculations, the following models are employed. The plasma is assumed to consist of electrons (e) and two heavy atomic species, ions (i) and radiators (r). The latter are representative of frequent cases when a tracer element, differing from the majority ions in the plasma by mass and/or charge, is used for spectroscopic diagnostics. Each of the species $s, s=\{\mathrm{e}, \mathrm{i}, \mathrm{r}\}$ may have different temperature $T_{s}$. The masses $m_{s}$ and charges $q_{s}$ of the heavy species $s=\{\mathrm{i}, \mathrm{r}\}$ are expressed in units of the proton mass $m_{\mathrm{p}}$ and elementary charge $e$, respectively:

$$
\begin{aligned}
m_{s} & =M_{s} m_{\mathrm{p}}, \\
q_{s} & =Z_{s} e .
\end{aligned}
$$

The density of the radiator species $N_{\mathrm{r}}$ in the plasma is determined by its fraction $0 \leq P_{\mathrm{r}} \leq 1$ relative to the total density of the heavy species:

$$
P_{\mathrm{r}}=N_{\mathrm{r}} /\left(N_{\mathrm{i}}+N_{\mathrm{r}}\right)
$$

Using the quasi-neutrality condition

$$
N_{\mathrm{e}}=Z_{\mathrm{i}} N_{\mathrm{i}}+Z_{\mathrm{r}} N_{\mathrm{r}},
$$

the ion and radiator densities are calculated as

$$
N_{\mathrm{i}}=\frac{1-P_{\mathrm{r}}}{Z_{\mathrm{i}}\left(1-P_{\mathrm{r}}\right)+Z_{\mathrm{r}} P_{\mathrm{r}}} N_{\mathrm{e}}
$$

and

$$
N_{\mathrm{r}}=\frac{P_{\mathrm{r}}}{Z_{\mathrm{i}}\left(1-P_{\mathrm{r}}\right)+Z_{\mathrm{r}} P_{\mathrm{r}}} N_{\mathrm{e}}
$$

respectively. All plasma particles are non-relativistic, i.e.,

$$
k_{\mathrm{B}} T_{s} \ll m_{s} c^{2},
$$

where $k_{\mathrm{B}}$ is the Boltzmann constant and $c$ is the speed of light.

The radiators are assumed to be hydrogen-like, i.e., a non-relativistic atom with a single electron above a core with

$$
Z_{\text {core }}=Z_{\mathrm{r}}+1 \text {. }
$$

The radiative transition of interest is defined by the upper $n_{u}$ and lower $n_{\ell}$ principal quantum numbers.

A macroscopic magnetic field $B$ is assumed to be homogeneous in space and constant in time.

\section{Formulary}

\subsection{Atomic units}

All calculations are performed internally using the atomic units (a.u.), i.e., the electron mass $m_{\mathrm{e}}$, the elementary charge $e$, the reduced Plank constant $\hbar$, and the Coulomb constant $1 /\left(4 \pi \varepsilon_{0}\right)$ are all assumed unity. Furthermore, the Boltzmann constant $k_{\mathrm{B}}$ is also assumed unity, thus, temperature is represented in energy units. We note that in the a.u. the speed of light $c$ is equal to the reciprocal value of the fine-structure constant $\alpha \approx 1 / 137$. 


\subsection{Basic entities}

In the following equations, the $s$ index stands for species and assumes, according to the model, values of e (electrons), i (ions) or $r$ (radiators).

A typical inter-particle distance (Wigner-Seitz radius):

$$
r_{s}=\sqrt[3]{\frac{3}{4 \pi N_{s}}}
$$

Mass density:

$$
\rho_{s}=m_{s} N_{s} .
$$

Total mass density:

$$
\rho_{\mathrm{tot}}=\sum_{s} \rho_{s}
$$

Debye length:

$$
\lambda_{\mathrm{D}, s}=\sqrt{\frac{T_{s}}{4 \pi N_{s} q_{s}^{2}}} .
$$

Full Debye length (screening by all plasma species not heavier than a given one):

$$
\bar{\lambda}_{\mathrm{D}, s}=\left(\sum_{m_{s}^{\prime} \leq m_{s}} \lambda_{\mathrm{D}, s^{\prime}}^{-1}\right)^{-1} .
$$

Coupling parameter:

$$
\Gamma_{s}=\frac{q_{s}^{2}}{r_{s} T_{s}}=\frac{1}{3}\left(\frac{r_{s}}{\lambda_{\mathrm{D}, s}}\right)^{2}
$$

Pressure (an ideal gas):

$$
p_{s}=N_{s} T_{s} .
$$

Total gas dynamics pressure:

$$
p_{\text {tot }}=\sum_{s} p_{s}
$$

Magnetic-field pressure:

$$
p_{B}=\frac{B^{2}}{8 \pi} \text {. }
$$

Beta parameter:

$$
\beta=\frac{p_{\text {tot }}}{p_{B}} .
$$

Thermal velocity:

$$
v_{\mathrm{th}, s}=\sqrt{T_{s} / m_{s}} .
$$

Gyrofrequency:

$$
\omega_{\mathrm{g}, s}=2 \pi f_{\mathrm{g}, s}=\frac{q_{s} B}{m_{s} c} .
$$

Gyroradius:

$$
r_{\mathrm{g}, s}=\frac{v_{\mathrm{th}, s}}{\omega_{\mathrm{g}, s}} .
$$


Classical distance of minimum approach:

$$
r_{\mathrm{m}, s}=q_{s}^{2} / T_{s}
$$

Thermal de Broglie wavelength:

$$
\lambda_{s}=\sqrt{\frac{2 \pi}{m_{s} T_{s}}} .
$$

Fermi energy of an ideal electron gas:

$$
E_{\mathrm{F}}=\frac{1}{2}\left(3 \pi^{2} N_{\mathrm{e}}\right)^{2 / 3}
$$

Chemical potential of an ideal electron gas:

$$
\mu_{\mathrm{e}}=E_{\mathrm{F}} \zeta\left(T_{\mathrm{e}} / E_{\mathrm{F}}\right)
$$

where the reduced Fermi-Dirac chemical potential $\zeta$ is approximately calculated as

$$
\zeta(t) \approx \begin{cases}1-\frac{\pi t}{12}-\frac{(\pi t)^{2}}{80} & t<0.3 \\ t\left[\ln \frac{4}{3 \sqrt{\pi} t^{3 / 2}}+\frac{\sqrt{2}}{3 \sqrt{\pi} t^{3 / 2}}+\cdots\right] & t \geq 0.3\end{cases}
$$

Here, the high- $t$ expansion in powers of $t^{-3 / 2}$ continues up to the $\sim 1 / t^{6}$ term [6], ${ }^{1}$ providing a better-than- $1 \%$ accuracy.

Chemical potential of an ideal classical gas (used for the heavy plasma particles, i.e., for ions and radiators, $s=\{\mathrm{i}, \mathrm{r}\})$ :

$$
\mu_{s}=T_{s} \ln \left(N_{s} \lambda_{s}^{3}\right)
$$

\subsection{Waves}

Plasma frequency:

$$
\omega_{\mathrm{p}, s}=2 \pi f_{\mathrm{p}, s}=\sqrt{\frac{4 \pi N_{s} q_{s}^{2}}{m_{s}}}
$$

Plasmon energy:

$$
E_{\mathrm{p}}=\omega_{\mathrm{p}, \mathrm{e}}
$$

Inertial length:

$$
\lambda_{\mathrm{i}, s}=c / \omega_{\mathrm{p}, s} .
$$

Alfvén velocity:

$$
v_{\mathrm{A}}=\left(\sum_{q_{s} \neq 0} \frac{\sqrt{4 \pi m_{S} N_{s}}}{B}\right)^{-1} .
$$

\footnotetext{
${ }^{1}$ Note that the leading " + " in the unnumbered expression following eq. (3) should be replaced with " $t$ ",
} 


\subsection{Collisions}

Thomson cross-section:

$$
\sigma_{\mathrm{T}, s}=\frac{8 \pi}{3} r_{0, s}^{2},
$$

where $r_{0, s}$ is the classical electromagnetic radius:

$$
r_{0, s}=\frac{\alpha^{2} q_{s}^{2}}{m_{s}} .
$$

Coulomb logarithm of species $s$ scattered off species $s^{\prime}$ :

$$
\lambda_{s \mid s^{\prime}}=\ln \frac{b_{\max }}{b_{\min }},
$$

where

$$
b_{\max }=\bar{\lambda}_{\mathrm{D}, s}+\bar{\lambda}_{\mathrm{D}, s^{\prime}}
$$

and

$$
b_{\min }=\frac{2\left|q_{s} q_{s^{\prime}}\right|\left(m_{s}+m_{s^{\prime}}\right)}{3 m_{s} m_{s^{\prime}}\left(v_{\mathrm{th}, s}+v_{\mathrm{th}, s^{\prime}}\right)^{2}} .
$$

Thermal relaxation rate of species $s$ due to collisions with species $s^{\prime}$ :

$$
v_{s \mid s^{\prime}}=\frac{8 \sqrt{2 \pi} q_{s}^{2} q_{s^{\prime}}^{2} n_{s^{\prime}} \lambda_{s \mid s^{\prime}}}{3 m_{s} m_{s^{\prime}}\left(v_{\mathrm{th}, s}^{2}+v_{\mathrm{th}, s^{\prime}}^{2}\right)^{3 / 2}} .
$$

Dreicer field:

$$
F_{\mathrm{D}}=\frac{4 \pi N_{\mathrm{e}} \lambda_{\mathrm{e} \mid \mathrm{e}}}{T_{\mathrm{e}}} .
$$

\subsection{Spectroscopy}

Transition energy:

$$
E_{\mathrm{tr}}=E_{u}-E_{\ell},
$$

where $E_{u}$ are $E_{\ell}$ bound energies of the upper and lower levels, respectively, calculated accounting for isotopic shift:

$$
E_{i}=\frac{Z_{\text {core }}^{2}}{2 n_{i}^{2}\left(1+1 / m_{\mathrm{r}}\right)} .
$$

Transition frequency:

$$
\omega_{\mathrm{tr}}=2 \pi f_{\mathrm{tr}}=E_{\mathrm{tr}} .
$$

Transition wavelength:

$$
\lambda_{\text {tr }}=c / f_{\text {tr }} .
$$

Minimal energy distance (distance to the nearest energy level):

$$
\left|E_{n_{u}}-E_{n_{u}+1}\right| .
$$


Transition oscillator strength for absorption is calculated exactly for the Lyman series $\left(n_{\ell}=1\right)$ and approximately using the quasi-classical Kramers formula with the Gaunt-factor correction $g$ [7]:

$$
f_{n_{\ell}, n_{u}}=\left\{\begin{array}{ll}
\frac{2^{8} n_{u}^{5}\left(n_{u}-1\right)^{2 n_{u}-4}}{3\left(n_{u}+1\right)^{2 n_{u}+4}} & n_{\ell}=1 \\
\frac{2^{5}}{3 \sqrt{3} \pi} \frac{1}{1-\left(n_{u} / n_{\ell}\right)^{2}} \frac{1}{n_{\ell}^{3}} \frac{1}{n_{u}^{3}} g & n_{\ell}>1
\end{array},\right.
$$

where

$$
g \approx 1-0.1728 \frac{1+\left(n_{u} / n_{\ell}\right)^{2}}{\left[\left(1-\left(n_{u} / n_{\ell}\right)^{2}\right) n_{\ell}\right]^{2 / 3}} .
$$

The Einstein coefficient $A$ (rate of the spontaneous emission):

$$
A_{n_{u}, n_{\ell}}=2 \alpha^{3} E_{\mathrm{tr}}^{2} f_{n_{\ell}, n_{u}} \frac{n_{\ell}^{2}}{n_{u}^{2}}
$$

Natural line-width:

$$
\Gamma_{\text {nat }}=\Gamma_{n_{u}}+\Gamma_{n_{\ell}},
$$

where only contributions due to the radiative decay to the ground state are retained:

$$
\Gamma_{n} \approx A_{n, 1}
$$

Fine structure is a sum of fine-structure splittings of the upper and lower levels:

$$
\Delta E_{\mathrm{fs}}=\Delta E_{n_{u}}+\Delta E_{n_{\ell}}
$$

where each is estimated as a difference between the fine-structure corrections of the levels with the lowest $(j=1 / 2)$ and the highest $(j=n-1 / 2)$ total angular momentum $j$ :

$$
\Delta E_{n}=\Delta E_{n, 1 / 2}-\Delta E_{n, n-1 / 2}
$$

where

$$
\Delta E_{n, j}=\frac{Z_{\mathrm{core}}^{4} \alpha^{2}}{2 n^{3}}\left(\frac{1}{j+1 / 2}-\frac{3}{4 n}\right)
$$

(see, e.g., [8]).

Doppler full width at half-maximum (FWHM, hereafter simply "width"):

$$
w_{\mathrm{D}}=\sqrt{8 \ln 2} \frac{v_{\mathrm{th}, \mathrm{r}}}{c} E_{\mathrm{tr}} .
$$

Zeeman splitting is estimated in the Paschen-Back limit according to

$$
\Delta E_{\mathrm{mf}}=2 \mu_{B} B,
$$

where $\mu_{B}$ is the Bohr magneton.

Classical ionization field (for the upper level):

$$
F_{\mathrm{i}}=\frac{Z_{\mathrm{core}}^{3}}{16 n_{u}^{4}} .
$$


Inglis-Teller field (the field at which the adjacent $n_{u}$ and $n_{u}+1$ Stark manifolds start to overlap):

$$
F_{\mathrm{IT}}=\frac{Z_{\mathrm{core}}^{3}\left(2 n_{u}+1\right)}{6 n_{u}^{4}\left(n_{u}+1\right)^{2}} .
$$

Typical Debye screening (plasma screening of a Coulomb field exerted by a probe charge at the distance of the Wigner-Seitz radius):

$$
\left(1+r_{s} / \lambda_{\mathrm{D}, s}\right) \exp \left(-r_{s} / \lambda_{\mathrm{D}, s}\right) .
$$

Holtsmark field [9]:

$$
F_{0, s}=2 \pi\left(\frac{4}{15}\right)^{2 / 3}\left|q_{s}\right| N_{s}^{2 / 3}
$$

Typical micro-field frequency:

$$
f_{F, s}=\sqrt{v_{\mathrm{th}, s}^{2}+v_{\mathrm{th}, \mathrm{r}}^{2}} / r_{s} .
$$

The Stark broadening is calculated in the quasi-contiguous (QC) approximation [10], applicable to transitions between degenerate atomic levels. The method is summarized below.

The quasi-static Stark width due to micro-fields of species $s$ is given by

$$
w_{\mathrm{qs}, s}=\bar{\omega}_{1 / 2}^{0} \frac{3\left(n_{u}^{2}-n_{\ell}^{2}\right)}{Z_{\mathrm{core}}} F_{0, s} \eta_{\mathrm{pp}, s} \eta_{\mathrm{rp}, s} \times\left\{\begin{array}{ll}
\frac{1}{2} & n_{u}-n_{\ell}=1 \\
1 & n_{u}-n_{\ell}>1
\end{array},\right.
$$

where $\bar{\omega}_{1 / 2}^{0} \approx 1.4385$, the perturber-perturber $\eta_{\mathrm{pp}, s}$ and radiator-perturber $\eta_{\mathrm{rp}, s}$ correlation correction factors are, respectively,

$$
\eta_{\mathrm{pp}, s}=\left(1+r_{s} / \bar{\lambda}_{\mathrm{D}, s}\right) \exp \left(-r_{s} / \bar{\lambda}_{\mathrm{D}, s}\right)
$$

and

$$
\eta_{\mathrm{rp}, s}=\exp \left(-q_{\mathrm{r}} q_{s} / r_{s} T_{s}\right) .
$$

(The $1 / 2$ factor in eq. (3.51), not present in the original study [10], was empirically found to serve as a rough correction for the $\Delta n=1$ case; we remind the reader that the QC approximation was derived assuming $\Delta n \gg 1$ ).

Static/dynamic Stark ratio:

$$
R_{s}=\frac{w_{\mathrm{qs}, s}}{f_{F, s} \eta_{\mathrm{rp}, s}} .
$$

Stark "quasistaticity" factor:

$$
\mathrm{f}_{s}=\frac{R_{s}}{R_{s}+0.5} .
$$

The Stark width due to species $s$ as perturbers:

$$
w_{\mathrm{S}, s}=\mathrm{f}_{s} w_{\mathrm{qs}, s},
$$

resulting in the total Stark width

$$
w_{\mathrm{S}, \mathrm{tot}}=\left[\sum_{s} w_{\mathrm{S}, s}{ }^{3 / 2}\right]^{2 / 3} .
$$




\subsection{Radiation}

Cyclotron losses [3] per plasma volume:

$$
-\frac{\mathrm{d} \mathcal{E}_{\mathrm{c}, s}}{\mathrm{~d} t}=\frac{4 \alpha^{3} q_{s}^{2} \omega_{\mathrm{g}, s}^{2} T_{s}}{3 m_{s}} N_{s} .
$$

Free-free (Bremsstrahlung) losses [3] per plasma volume:

$$
-\frac{\mathrm{d} \mathcal{E}_{\mathrm{ff}}}{\mathrm{d} t}=\frac{8 \pi \alpha^{3} \sqrt{T_{\mathrm{e}}} N_{\mathrm{e}}}{3} \sum_{s \neq \mathrm{e}} N_{s} q_{s}^{2}
$$

Free-bound (recombination) losses per plasma volume:

$$
-\frac{\mathrm{d} \mathcal{E}_{\mathrm{fb}}}{\mathrm{d} t}=\frac{4 \pi \alpha^{3} N_{\mathrm{e}}}{3 \sqrt{T_{\mathrm{e}}}} \sum_{s \neq \mathrm{e}} N_{s} q_{s}^{4} .
$$

The expression above is obtained using, for each heavy species $s$, the approximate relation [11] $\frac{\mathrm{d} \mathcal{E}_{\mathrm{ff}, s}}{\mathrm{~d} t} / \frac{\mathrm{d} \mathcal{E}_{\mathrm{ff}}, s}{\mathrm{~d} t} \approx \frac{E_{\infty, s}}{T_{\mathrm{e}}}$, where $E_{\infty, s}=q_{s}^{2} / 2$ is the bound energy of the ground state of the recombined ion.

The power spectral density of the free-free radiation at the transition energy $E_{\mathrm{tr}}$ :

$$
-\frac{\mathrm{d} \mathcal{E}_{\mathrm{ff}}\left(E_{\mathrm{tr}}\right)}{\mathrm{d} t}=-\frac{\exp \left(-E_{\mathrm{tr}} / T_{\mathrm{e}}\right)}{T_{\mathrm{e}}} \frac{\mathrm{d} \mathcal{E}_{\mathrm{ff}}}{\mathrm{d} t}
$$

and similarly for the free-bound radiation:

$$
-\frac{\mathrm{d} \mathcal{E}_{\mathrm{fb}}\left(E_{\mathrm{tr}}\right)}{\mathrm{d} t}=\frac{8 \pi \alpha^{3} N_{\mathrm{e}}}{3 T_{\mathrm{e}}^{3 / 2}} \sum_{s \neq \mathrm{e}} \theta\left(E_{\mathrm{tr}}-E_{\infty, s}\right) N_{s} q_{s}^{2} E_{\infty, s} \exp \left[-\left(E_{\mathrm{tr}}-E_{\infty, s}\right) / T_{\mathrm{e}}\right]
$$

where $\theta(x)$ is the Heaviside step function $(\theta(x)$ is zero for negative $x$ and unity for positive $x$ ). Evidently, only recombination to the ground state is accounted for.

\section{User's guide}

"Plasma Formulary Interactive" is built as a JavaScript Web application in order to provide a maximal cross-platform capability, independent of an underlying operating system. The graphical user interface (GUI) is built using the Qooxdoo toolkit [12] and should be compatible with a majority of modern browsers. The required resources are rather moderate, making the application responsive even on low-end devices such as modern smartphones. It is available at the following address: http://plasma-gate.weizmann.ac.il/pf/.

The main GUI consists of four tabbed panels. The first, "Parameters" panel (see figure 1) allows for defining the parameters of the plasma model (section 2): $N_{\mathrm{e}}, T_{\mathrm{e}}, Z_{\mathrm{i}}, M_{\mathrm{i}}, T_{\mathrm{i}}, Z_{\mathrm{r}}, M_{\mathrm{r}}, T_{\mathrm{r}}$, $P_{\mathrm{r}}, B$, and those of the atomic system of the radiator: $n_{u}$ and $n_{\ell} . N_{\mathrm{i}}, N_{\mathrm{r}}$, and $Z_{\text {core }}$ are automatically updated using eqs. (2.5), (2.6), and (2.8), respectively. Since usually one is interested in fully thermalized plasmas $\left(T_{\mathrm{e}}=T_{\mathrm{i}}=T_{\mathrm{r}}\right)$, for convenience, changing $T_{\mathrm{e}}$ automatically updates $T_{\mathrm{i}}$ and $T_{\mathrm{r}}$, while changing $T_{\mathrm{i}}$ automatically updates $T_{\mathrm{r}}$. Therefore, if $T_{\mathrm{e}} \neq T_{\mathrm{i}} \neq T_{\mathrm{r}}$ is required, one should first 


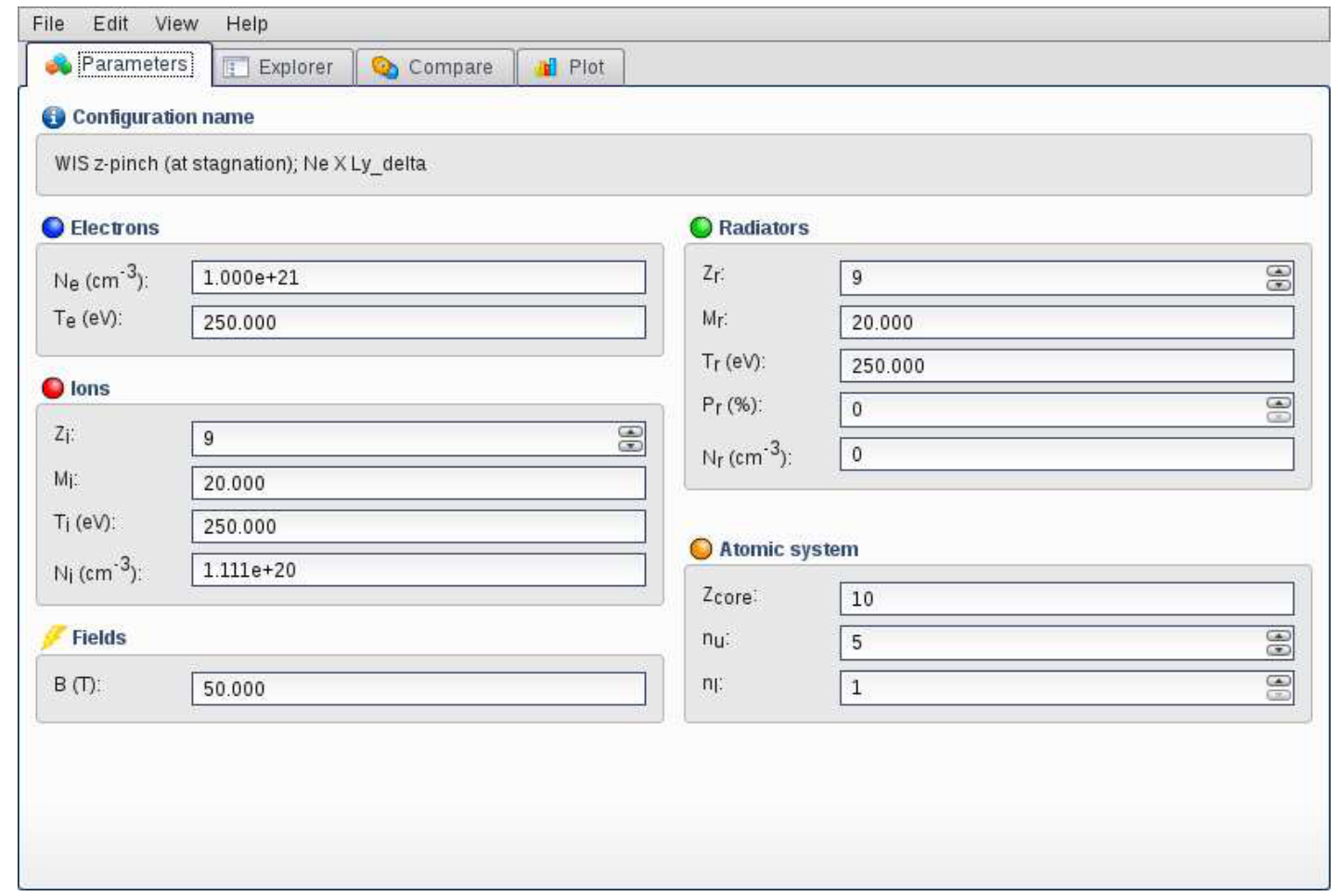

Figure 1. (Color online) The "Parameters" panel.

enter $T_{\mathrm{e}}$, followed by $T_{\mathrm{i}}$ and further followed by setting $T_{\mathrm{r}}$. A complete set of plasma parameters can be saved using "File $\rightarrow$ Save" or "File $\rightarrow$ Save As" menu entries under a desired name for further loading.

The next, "Explorer" panel (figure 2), allows for browsing plasma entities arranged in a tree structure according to the dimension units. The complete list is given below, alongside with references to the corresponding expressions in parentheses.

- Dimensionless

- Coupling parameter (3.6)

- Typical Debye screening (3.48)

- Beta (3.10)

- Oscillator strength (3.36)

- Stark quasistaticity (3.53)

- Static/dynamic Stark ratio (3.52)

- Coulomb logarithm (3.26)

- Length

- Wigner-Seitz radius (3.1)

- Debye length (3.4)

- Full Debye length (3.5)

- Gyroradius (3.13)

- Distance of minimal approach (3.14)

- Inertial length (3.22) 


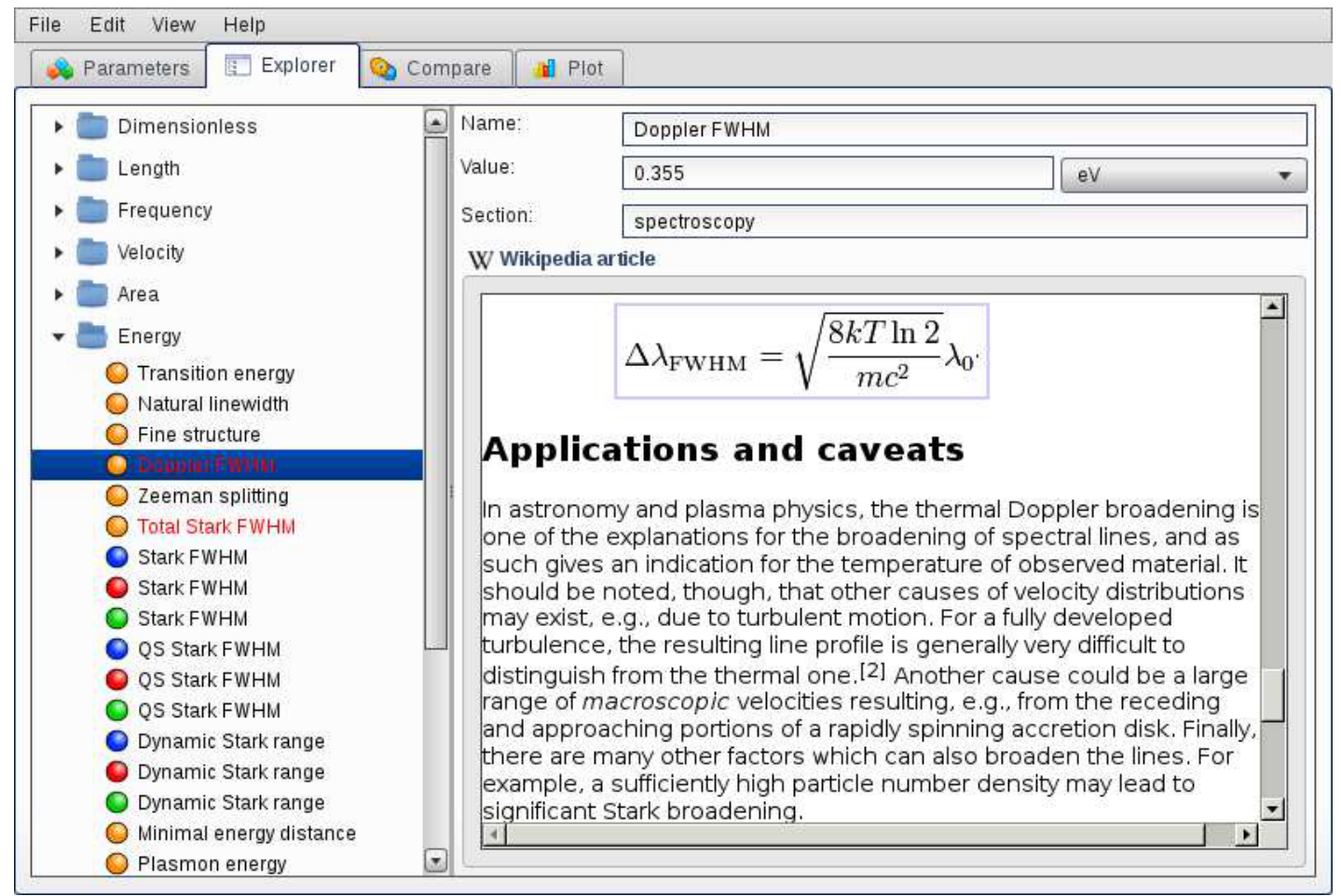

Figure 2. (Color online) The "Explorer" panel.

- De Broglie wavelength (3.15)

- Transition wavelength (3.34)

- Frequency

- Plasma frequency (3.20)

- Gyrofrequency (3.12)

- Transition frequency (3.33)

- Einstein coefficient (3.38)

- Microfield frequency (3.50)

- Relaxation rate (3.29)

- Velocity

- Thermal velocity (3.11)

- Alfvén velocity (3.23)

- Area

- Thomson cross-section (3.24)

- Mass density

- Mass density (3.2)

- Total mass density (3.3)

- Energy

- Transition energy (3.31)

- Natural linewidth (3.39)

- Fine structure (3.41) 
- Doppler FWHM (3.44)

- Zeeman splitting (3.45)

- Total Stark FWHM (3.55)

- Stark FWHM (3.54)

- QS Stark FWHM (3.51)

- Dynamic Stark range (3.50)

- Minimal energy distance (3.35)

- Plasmon energy (3.21)

- Fermi energy (3.16)

- Chemical potential (3.17) and (3.19)

- Pressure

- Ideal gas pressure (3.7)

- Total pressure (3.8)

- Magnetic field pressure (3.9)

- Power density

- Cyclotron losses (3.56)

- Free-free losses (3.57)

- Free-bound losses (3.58)

- Power spectral density

- Free-free spectral density (3.59)

- Free-bound spectral density (3.60)

- Electric field

- Holtsmark field (3.49)

- Classical ionization field (3.46)

- Inglis-Teller field (3.47)

- Dreicer field (3.30)

If desired, the displayed entities can be filtered either according to the species they refer to (Electrons/Ions/Radiators), or by section they belong to (Fundamental/Waves/Collisions/Spectroscopy/Radiation) using the "View $\rightarrow$ Show Species" and "View $\rightarrow$ Show Sections" menu entries, respectively.

Selecting an entity from the list results in its value being calculated (for the plasma parameters selected) and displayed, and the corresponding Wikipedia article (if exists) optionally shown. The calculated value can be shown in a.u., SI, CGS, and some more widely used units, e.g., angstroms ( $\AA$ ) for entities having dimension of length. While selected, the entity can be added to, or removed from, the list of "favorites" using either the context-sensitive or menubar "Edit" pull-down menu. The "favorite" entities are used for further comparison or plotting (see below).

The third, "Compare" panel (figure 3) serves for comparison of previously selected "favorite" entities, where these are listed in a table by groups having the same dimension.

Finally, the "Plot" panel, shown in figure 4, allows for graphing the previously selected "favorites" (grouped by the same dimension) as a function of one of the parameters of the plasma model: $N_{\mathrm{e}}, T_{\mathrm{e}}, T_{\mathrm{i}}, T_{\mathrm{r}}, P_{\mathrm{r}}$, and $B$. It is also possible to change $T_{\mathrm{i}}$ and $T_{\mathrm{r}}$ or all three $T_{\mathrm{e}}, T_{\mathrm{i}}$, and $T_{\mathrm{r}}$ simultaneously. The range of the parameter being varied, line colors, and number of points are adjustable. 


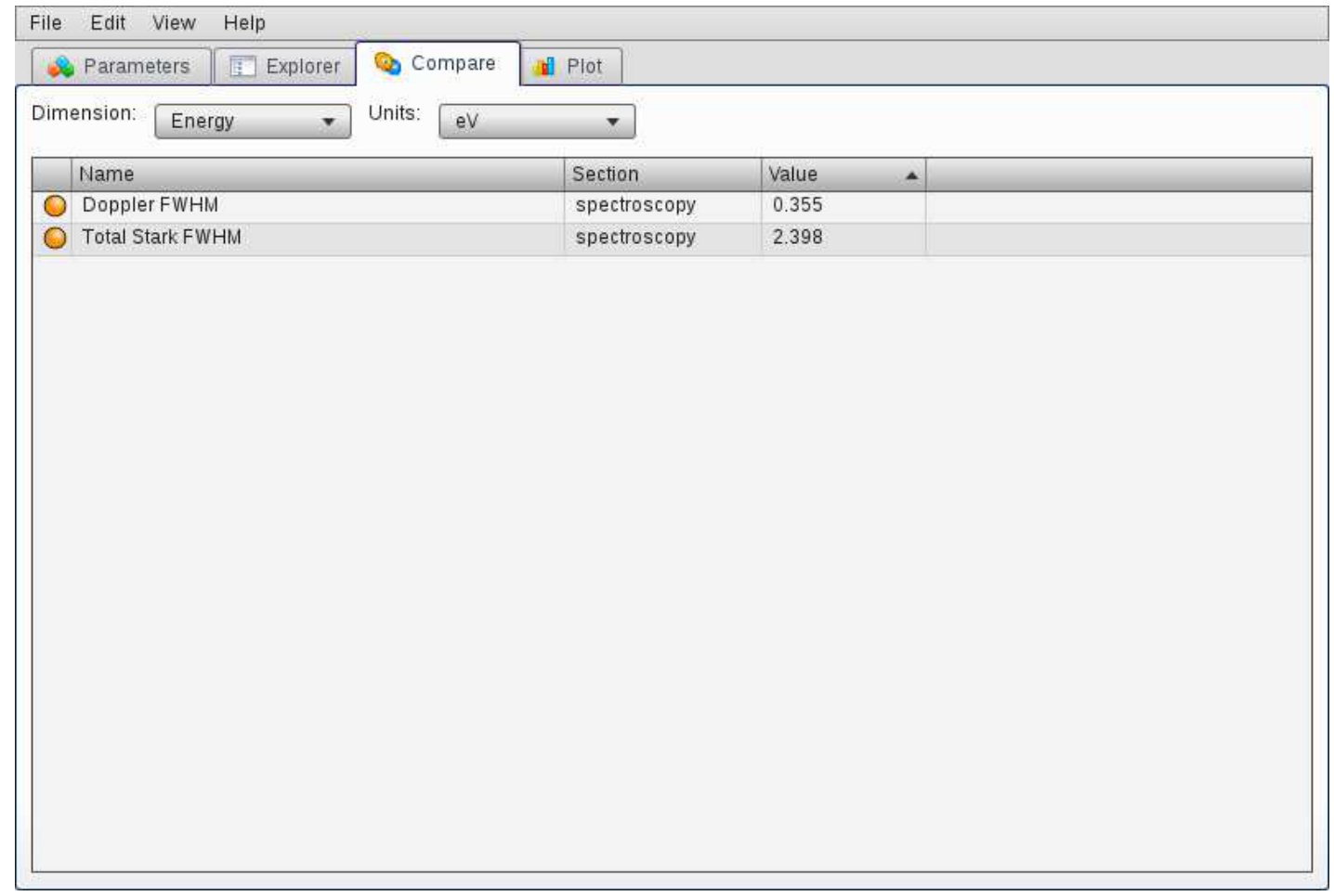

Figure 3. (Color online) The "Compare" panel.

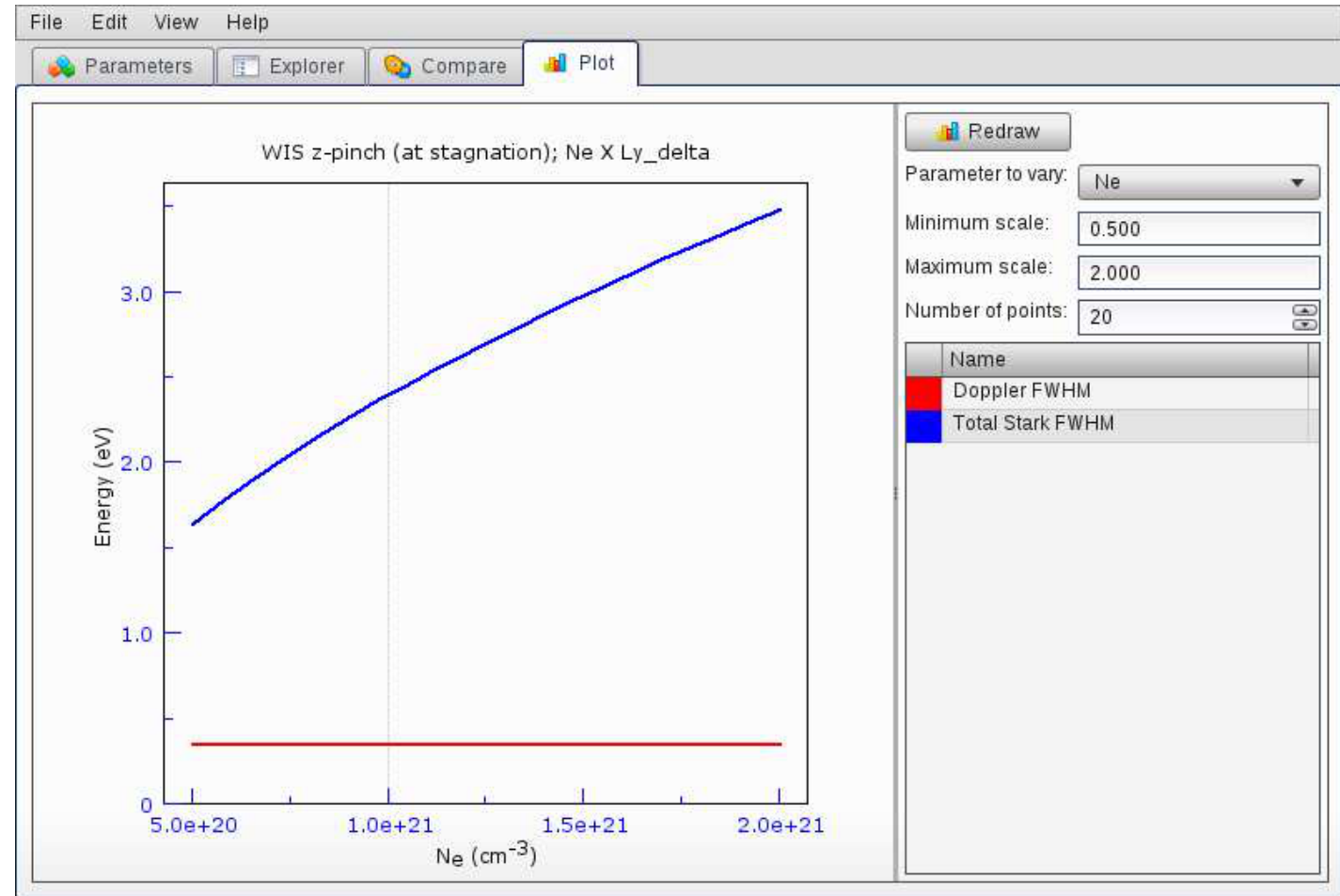

Figure 4. (Color online) The "Plot" panel. 


\section{Acknowledgments}

This work was supported in part by the Israel Science Foundation, the Naval Research Laboratory (USA), and the Rom Charitable Trust (USA).

\section{References}

[1] J.D. Huba, NRL plasma formulary, http://wwwppd.nrl .navy.mil/nrlf ormulary/, 2009.

[2] A. Anders, A Formulary for Plasma Physics, Akademie-Verlag, Berlin Germany (1990).

[3] D.A. Diver, Plasma Formulary for Physics, Technology and Astrophysics, Wiley-VCH, (2001).

[4] Free Software Foundation, Inc., GNU General Public License, http://www.gnu.org/licenses/gpl.html, 2007.

[5] Wikipedia, http://www.wikipedia.org.

[6] C. Ebner and H. Fu, Thermodynamic functions of ideal two- and three-dimensional Fermi gases, J. Low Temp. Phys. 16 (1974) 43.

[7] D.H. Menzel and C.L. Pekeris, Absorption coefficients and hydrogen line intensities, Mon. Not. Roy. Astron. Soc. 96 (1935) 77.

[8] H.A. Bethe and E.E. Salpeter, Quantum Mechanics of One- and Two-Electron Atoms, Plenum, New York U.S.A. (1977).

[9] J. Holtsmark, Über die verbreiterung von spektrallinien, Ann. Phys. 58 (1919) 577.

[10] E. Stambulchik and Y. Maron, Stark effect of high-n hydrogen-like transitions: quasi-contiguous approximation, J. Phys. B 41 (2008) 095703.

[11] H.R. Griem, Principles of Plasma Spectroscopy, Cambridge University Press, Cambridge U.K. (1997).

[12] 1\&1 Internet AG, Qooxdoo framework, http://qooxdoo.org/. 